\title{
Cáncer por contaminación química del agua de consumo humano en menores de 19 años: una revisión sistemática
}

\author{
Vanessa Oller-Arlandis ${ }^{1}$ y Javier Sanz-Valero ${ }^{1}$
}

Forma de citar

Oller-Arlandis V, Sanz-Valero J. Cáncer por contaminación química del agua de consumo humano en menores de 19 años: una revisión sistemática. Rev Panam Salud Publica. 2012;32(6):435-43.

RESUMEN Objetivo. Evaluar la asociación entre la exposición a los principales contaminantes químicos del agua de consumo humano $(A C H)$ y el aumento de casos de cáncer en menores de 19 años.

Métodos. Se realizó una revisión sistemática de la literatura científica recogida en las bases de datos MEDLINE (via PubMed ${ }^{\odot}$ ), EMBASE ${ }^{\odot}$, Web of Knowledge, Cochrane Library Plus, Literatura Latinoamericana y del Caribe en Ciencias de la Salud (LILACS), SCOPUS y SCIRUS. Los descriptores utilizados fueron "neoplasms" y "water pollution, chemical", limitado a estudios que incluyeran a menores de 19 años. Se seleccionaron los artículos científicos de cualquier tipo y en cualquier idioma, desde el inicio de la indización de la fuente primaria hasta marzo de 2011.

Resultados. Se recuperaron 266 artículos, de los que se seleccionaron 20 tras aplicar los criterios de inclusión y exclusión. Los contaminantes del ACH analizados fueron: arsénico, subproductos de la desinfección, compuestos nitrogenados, derivados del petróleo, plaguicidas agrícolas, radionúclidos, así como otros de origen industrial. La mayoría de los estudios no hallaron una asociación significativa entre la exposición a los contaminantes encontrados en el ACH y el aumento de casos de cáncer en menores de 19 años. En algunas de las poblaciones estudiadas se observó una relación significativa dosis-respuesta en el período de exposición.

Conclusiones. Teniendo en cuenta el insuficiente nivel de actualidad de los artículos encontrados, se requieren más estudios dirigidos a conocer el efecto real de la contaminación del ACH en la incidencia de cáncer en la población, en particular en los niños y jóvenes por su mayor susceptibilidad.

Palabras clave Neoplasias; contaminación química del agua; niño; adolescente; agua potable.

Desde la década de 1970, diversos estudios epidemiológicos han sugerido una asociación entre los contaminantes químicos con niveles superiores a los admisibles en el agua para consumo humano $(\mathrm{ACH})$ y la mayor probabilidad de desarrollar algún tipo de cáncer (1-3).

\footnotetext{
1 Departamento de Salud Pública e Historia de la Ciencia y Ginecología, Universidad Miguel Hernández, Valencia, España. La correspondencia se debe enviar a Javier Sanz-Valero. Correo electrónico: jsanz@umh.es
}

Además, los niños menores de 2 años representan una subpoblación especialmente vulnerable a las sustancias carcinógenas, ya que su riesgo de desarrollar neoplasias malignas por esta causa es 10 veces mayor que el de los adultos y 3 veces mayor que el de los adolescentes de 3 a 15 años (4).

$\mathrm{El}$ agua es un solvente excelente que puede contener en disolución un gran número de sustancias carcinógenas, como el arsénico, clasificado por la Agencia Inter- nacional de Investigación sobre Cáncer como un posible carcinógeno para el ser humano (5) y que podría estar asociado con el cáncer de piel, hígado, pulmón o vejiga $(6,7)$.

Por otra parte, los subproductos resultantes de los tratamientos de desinfección del agua, generados por la reacción del desinfectante con la materia orgánica, son también contaminantes potenciales del ACH (8). El uso de cloro, uno de los desinfectantes más utilizados, produce 
trihalometanos — como el cloroformo y el bromodiclorometano-, sustancias clasificadas entre las posibles causantes de leucemias $(2,3,5,6)$.

El ACH también puede contener nitratos y nitritos, precursores endógenos de compuestos nitrogenados que pueden producir efectos adversos a la salud de los niños, incluido el cáncer de estómago y tumores cerebrales y del sistema nervioso $(6,9)$. Otros compuestos orgánicos volátiles, hidrocarburos policíclicos $\mathrm{y}$ metales procedentes de diversas fuentes de contaminación, como la agricultura, los vertimientos industriales y la lixiviación de minerales, también contaminan las aguas subterráneas y se han asociado con las leucemias $(6,10)$.

En este trabajo se evalúa, mediante una revisión sistemática, la asociación entre la exposición a los principales contaminantes químicos del ACH y el aumento de casos de cáncer en menores de 19 años.

\section{MATERIALES Y MÉTODOS}

Se realizó una revisión sistemática de la literatura científica recogida en las bases de datos MEDLINE (via PubMed ${ }^{\odot}$ ), EMBASE $^{\odot}$, Web of Knowledge (Institute for Scientific Information), Cochrane Library Plus, Literatura Latinoamericana y del Caribe en Ciencias de la Salud (LILACS), SCOPUS y SCIRUS.

Se buscaron los artículos publicados en cualquier país, por cualquier institución y en cualquier idioma, desde el inicio de la indización de cada una de las fuentes primarias hasta marzo de 2011. Para la recuperación documental se emplearon los términos $\mathrm{MeSH}(\mathrm{Me}-$ dical Subject Headings) "neoplasms" y "water pollution, chemical" con el límite "humanos", sin calificadores de ningún tipo. En MEDLINE se utilizaron los conectores booleanos, y se adaptaron posteriormente a las otras bases de datos.

Se buscaron todos los artículos originales publicados en revistas arbitradas, cuya población de estudio incluyera a menores de 19 años (niños y adolescentes) y que trataran sobre agua contaminada por compuestos químicos y su relación con cualquier tipo de neoplasia maligna. No se establecieron restricciones en cuanto al sexo de los participantes o el tipo de muestra.

Se excluyeron los artículos cuyo texto completo no se pudo recuperar y los que no se centraron en humanos o en el cáncer infantil, ya sea que estudiaran otras enfermedades (neuropatía periférica, síntomas gastrointestinales, diabetes, enfermedades cardiovasculares, etc.) $\mathrm{u}$ otros efectos (por ejemplo, mutaciones genéticas, daños cromosómicos inducidos por factores químicos, etc.) Igualmente, se eliminaron los artículos cuya vía de exposición al contaminante no era el ACH (aire, alimentos, etc.)

Con el objetivo de reducir el sesgo de publicación, se incluyeron en el análisis otros estudios encontrados en las referencias bibliográficas de los artículos seleccionados y no detectados en la búsqueda inicial.

La pertinencia de los artículos se evaluó de forma independiente por los dos autores. Como soporte en la evaluación de la calidad de los artículos se siguieron las recomendaciones de STROBE (Strengthening the Reporting of Observational Studies in Epidemiology) (11). Para dar por válida la selección de los artículos a considerar se estableció que la fuerza de la concordancia (índice kappa) entre los dos autores debía ser superior a 0,60 (buena o muy buena) (12). Siempre que se cumpliera esta condición, las posibles discordancias se solucionarían mediante el consenso entre los autores.

El control de la calidad de la información se realizó mediante tablas de doble entrada que permitían la detección de los errores y su corrección mediante una nueva consulta de los originales.

Para la extracción de los datos, los estudios se agruparon por el tipo de contaminante químico presente en el $\mathrm{ACH}$. Los datos más relevantes de cada trabajo (autores, año de publicación, diseño, forma de exposición, tipo de cáncer, población estudiada, período de seguimiento, lugar donde se efectuó la exposición y los principales resultados) se resumieron en un cuadro.

Para determinar la actualidad u obsolescencia de los artículos se utilizaron dos indicadores bibliométricos: el semiperíodo de Burton-Kebler (la mediana de la antigüedad de las referencias) y el índice de Price (porcentaje de referencias con antigüedad igual o inferior a 5 años) (13).

\section{RESULTADOS}

De los 266 artículos recuperados, tras aplicar los criterios de inclusión y exclusión, se seleccionaron finalmente 20 artículos (14-33). Ningún artículo se rechazó por causas metodológicas (figura 1); todos los artículos seleccionados superaron la mediana de los criterios propuestos por STROBE para los estudios observacionales (34). A pesar de no haber discriminado por idioma, los artículos seleccionados estaban en inglés o español (cuadro 1).

No fue necesario valorar la concordancia entre los autores, ya que la coincidencia sobre la pertinencia de los trabajos fue de $100 \%$. Según los resultados del análisis de obsolescencia $(35,36)$, la actualidad de los trabajos recuperados fue baja (obsolescencia de 12 años; índice de Price de 10,53\%).

La información sobre la población estudiada y el grado de exposición a los contaminantes fue muy heterogénea: mientras en unos estudios se precisaba bien la edad, el sexo, el tiempo de exposición y la dosis de exposición $(16,28)$, en otros no se aportaban datos suficientes $(18,26)$. La mayor población estudiada fue de 3805745 nacimientos (14) y la menor, de 56 personas (20).

De los 20 artículos analizados, 10 se referían a estudios realizados en los Estados Unidos de América (14,17-21, 23, 26, 30, 33), 3 en Canadá $(22,27,29), 2$ en el Reino Unido $(25,32)$ y 1 en cada uno de los siguientes países: Chile (15), Ecuador (24), India (16), Japón (28) y México (31). El estudio más prolongado duró 50 años y se llevó a cabo en Chile (15), mientras que el más breve duró 3 años y se realizó Bengala Occidental, India (16).

\section{El arsénico y otros metales como contaminantes del ACH}

Con respecto a los contaminantes químicos del ACH y sus valores máximos encontrados (cuadro 2), el más frecuentemente estudiado fue el arsénico $(15,16$, $18,20-22,28,31$ ).

En el estudio realizado en Chile (15) se evaluaron retrospectivamente los patrones de tiempo y la localización de la mortalidad por cáncer de hígado entre 1950 y 2000 en dos regiones con distintos períodos de exposición. Se concluyó que la exposición a elevados niveles de arsénico en el ACH (1950-1957 0,09 $\mathrm{mg} / \mathrm{L} ; 1958-1970=0,87 \mathrm{mg} / \mathrm{L})$ durante las primeras etapas de la infancia puede dar lugar al incremento de la mortalidad por cáncer hepático. Sin embargo, no se encontró que aumentara la mortalidad 
FIGURA 1. Diagrama del proceso de selección de artículos

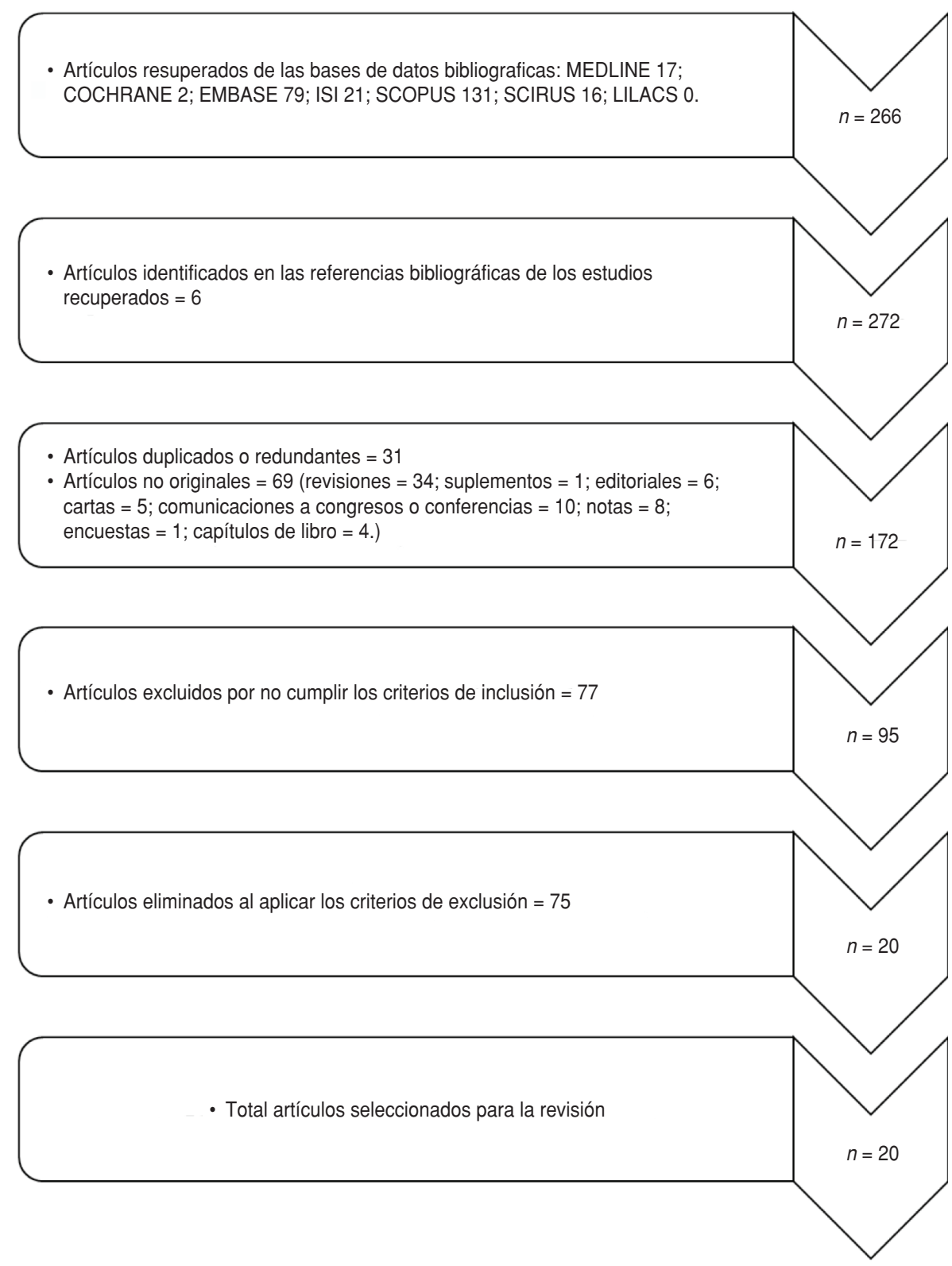

por otras neoplasias malignas infantiles en la región de exposición respecto a la de referencia.

En Bengala Occidental, India, un estudio de cohortes (16) reveló que ningún niño expuesto a concentraciones de arsénico de 0,92 $\mathrm{mg} / \mathrm{L}$ presentaba cáncer de piel, aunque sí se observaron lesiones cutáneas.

En un intento de explicar un conglomerado de casos de leucemia infantil en Fallon, Estados Unidos, se midieron los niveles de contaminación del agua de 100 pozos y se detectó que tenían elevados niveles de arsénico (18). Sin embargo, la
También se encontró arsénico en el ACH (0,04-0,09 mg/L) en un estudio ecológico llevado a cabo en Nevada, Estados Unidos (21), y en otro de casos y controles realizado en Quebec, Canadá, $(<0,01 \mathrm{mg} / \mathrm{L})(22)$ —que evaluó también la exposición a cadmio $(<0,01 \mathrm{mg} /)$, cromo $(<0,01 \mathrm{mg} / \mathrm{L})$, plomo $(0,01 \mathrm{mg} / \mathrm{L})$ y zinc $(17,10 \mathrm{mg} / \mathrm{L})$-; sin embargo, no encontraron ninguna asociación significativa con el aumento de casos de leucemia en la infancia.

En un estudio de cohortes realizado en Japón (28) se encontró una asociación significativa entre la exposición a concentraciones de arsénico superiores a $1 \mathrm{mg} / \mathrm{L}$ y la mortalidad por cáncer de pulmón y de tracto urinario, tras un seguimiento de 33 años. Otro trabajo llevado a cabo en una comunidad rural de México (31) reveló la aparición de un carcinoma epidermoide y cirrosis hepática en un niño de 13 años que había consumido ACH contaminada con arsénico (10 mg/L) durante 7 años.

\section{Subproductos de la desinfección y compuestos nitrogenados}

En Quebec, Canadá, un estudio de casos y controles (22) que evaluó el ACH como vía de exposición a los subproductos de desinfección - trihalometanos totales y específicos- no encontró datos que respaldaran una asociación significativa con el aumento de casos de leucemia en niños de hasta 9 años.

Por su parte, un trabajo desarrollado en Maryland, Estados Unidos (17), encontró una posible asociación significativa entre la exposición a nitratos y la leucemia infantil. En Canadá (22), una investigación que evaluó la exposición a nitratos $(1,99 \mathrm{mg} / \mathrm{L})$ y el riesgo de leucemia infantil tampoco encontró una asociación significativa.

Otro trabajo (23), que estudió la relación del cáncer cerebral en niños con los niveles de iones nitrato y nitrito en los suministros de $\mathrm{ACH}$ de tres condados estadounidenses, no encontró una asociación entre los niveles a los que se expusieron las mujeres embarazadas y el riesgo de que sus hijos sufrieran esa enfermedad. Sin embargo, las mujeres residentes en el oeste del estado de Washington - uno de los tres centros de estudio-, que utilizaron pozos privados como fuente de $\mathrm{ACH}$ durante el embarazo, mostraron un riesgo significativa- 







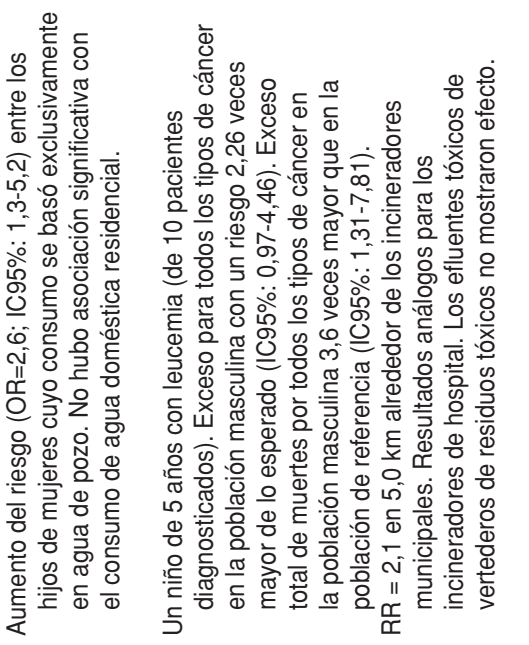

密

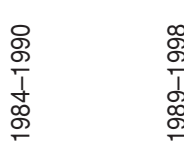

응을

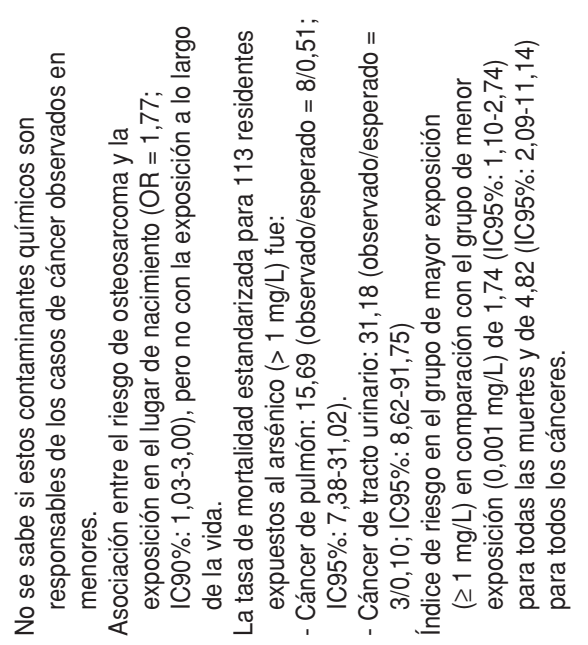

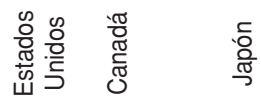

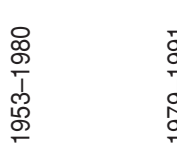

용. 을

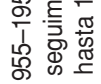

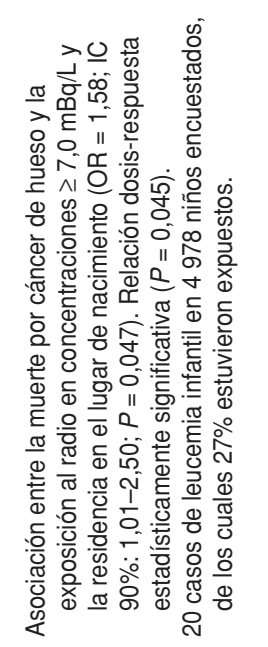

$\frac{\pi}{\pi} \underset{\pi}{\pi}$

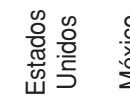

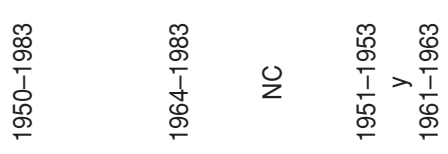

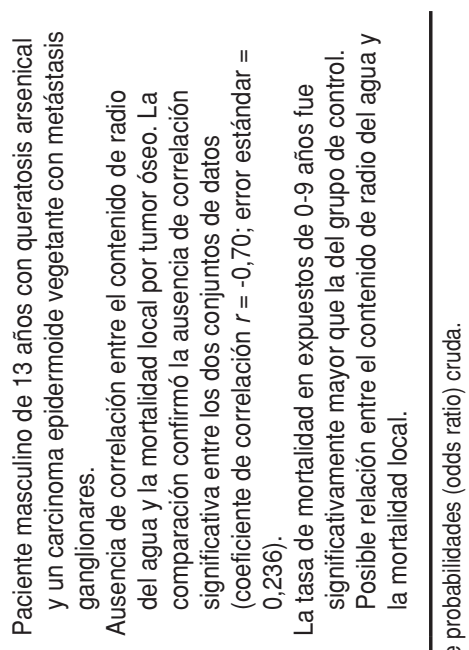

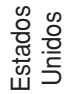

兽

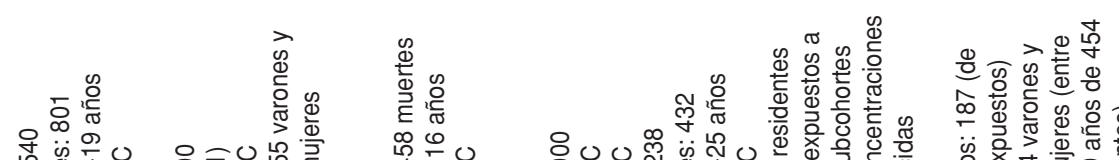

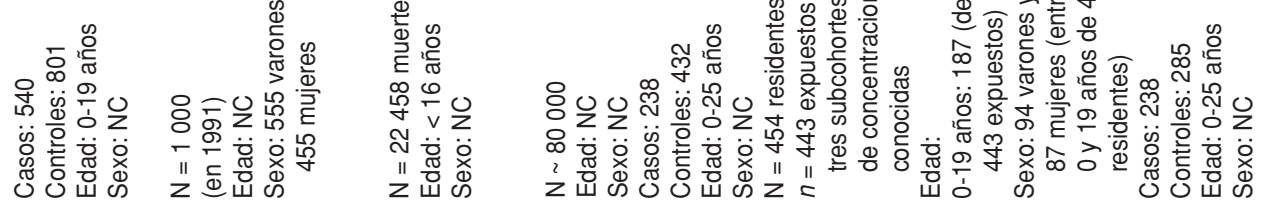

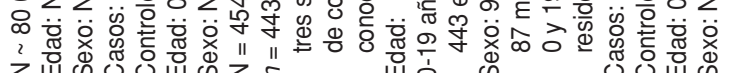

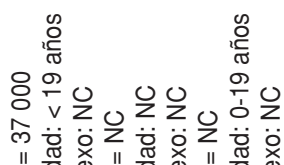

융융

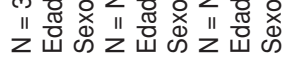
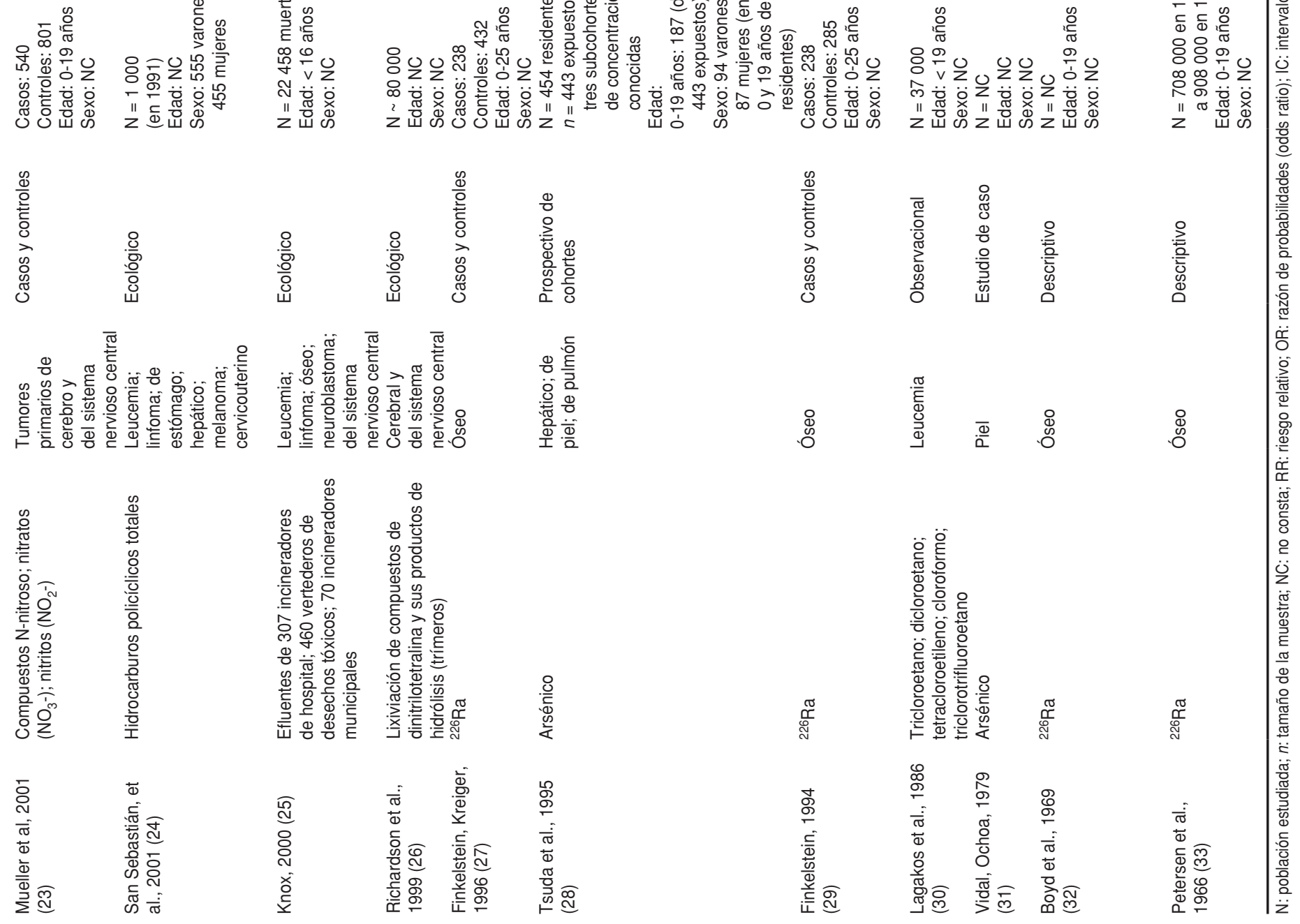
CUADRO 2. Niveles máximos permitidos en el agua de consumo humano según tres diferentes normas $^{\mathrm{a}}$

\begin{tabular}{|c|c|c|c|}
\hline Compuesto & $\begin{array}{l}\text { Organización Mundial } \\
\text { de la Salud }\end{array}$ & Unión Europea & Estados Unidos \\
\hline \multicolumn{4}{|l|}{ Elementos } \\
\hline Arsénico & 0,010 & 0,010 & 0,010 \\
\hline Cadmio & 0,003 & 0,005 & 0,005 \\
\hline Cromo & $0,05(p)$ & 0,050 & 0,1 \\
\hline Níquel & 0,07 & 0,020 & ND \\
\hline Plomo & 0,01 & 0,010 & 0,015 \\
\hline Zinc & $3^{b}(p)$ & ND & $5^{c}$ \\
\hline \multicolumn{4}{|l|}{ Compuestos inorgánicos } \\
\hline Nitratos & $\begin{array}{c}50 \text { (como } \mathrm{NO}_{3}^{-} \text {) } \\
11 \text { (como nitrato-nitrógeno) }\end{array}$ & $\begin{array}{c}50 \\
\text { (como } \mathrm{NO}_{3}^{-} \text {) }\end{array}$ & $\begin{array}{c}10 \\
\text { (como nitrato-nitrógeno) }\end{array}$ \\
\hline Nitritos & $\begin{array}{c}\left.3 \text { (como } \mathrm{NO}_{2}^{-}\right) \\
0,9 \text { (como nitrito-nitrógeno) }\end{array}$ & $\left.\begin{array}{c}0,50 \\
(\text { como NO} \\
-\end{array}\right)$ & $\begin{array}{c}1 \\
\text { (como nitrito-nitrógeno) }\end{array}$ \\
\hline \multicolumn{4}{|l|}{ Compuestos orgánicos } \\
\hline Atrazina & 0,1 & $0,00010^{d}$ & 0,003 \\
\hline Benzeno & 0,01 & 0,001 & 0,005 \\
\hline Bromodiclorometano & 0,06 & ND & 0 \\
\hline Bromoformo & 0,1 & ND & 0 \\
\hline Cloroformo & 0,3 & ND & 0,07 \\
\hline Dibromoclorometano & 0,1 & ND & 0,06 \\
\hline 1,2-Dicloroetano & 0,03 & 0,003 & 0,005 \\
\hline $\begin{array}{l}\text { Hidrocarburos policíclicos } \\
\text { aromáticos }\end{array}$ & ND & 0,0001 & 0,0002 \\
\hline Metolaclor & 0,01 & $0,00010^{d}$ & ND \\
\hline Tetracloroetileno & 0,04 & $0,005^{\mathrm{e}}$ & 0,005 \\
\hline Tricloroetileno & $0,02(p)$ & $0,005^{\mathrm{e}}$ & 0,005 \\
\hline Trihalometanos totales & $f$ & 0,100 & 0,080 \\
\hline \multicolumn{4}{|l|}{ Radionúclidos } \\
\hline Uranio & $0,03(p)$ & ND & 0,030 \\
\hline${ }^{226} \mathrm{Ra}$ & $1 \mathrm{~Bq} / \mathrm{L}^{\mathrm{g}}$ & ND & ND \\
\hline${ }^{228} \mathrm{Ra}$ & $0,1 \mathrm{~Bq} / \mathrm{Lg}$ & ND & ND \\
\hline${ }^{226} \mathrm{Ra}+{ }^{228} \mathrm{Ra}$ & ND & ND & $5 \mathrm{pCi} / \mathrm{L}^{\mathrm{h}}$ \\
\hline
\end{tabular}

ND: no hay datos disponibles en el documento normativo; (p): valor de referencia provisional, debido a que hay datos sobre la peligrosidad de la sustancia, pero es aún insuficiente la información disponible relativa a sus efectos sobre la salud.

a Todos los valores están expresados en $\mathrm{mg} / \mathrm{L}$, excepto los que se indican explícitamente con sus unidades.

${ }^{b}$ No implica problemas para la salud a los niveles normalmente hallados en el $\mathrm{ACH}$; sin embargo, niveles superiores pueden determinar la no aceptabilidad para el consumo.

c Según la lista secundaria de contaminantes del agua de bebida de la Agencia de Protección Ambiental de los Estados Unidos.

d Este parámetro se aplica a cada uno de los plaguicidas; la suma de las cantidades de todos los plaguicidas detectados y cuantificados en el procedimiento de control debe ser inferior a $0,0005 \mathrm{mg} / \mathrm{L}$.

e Para tetracloroeteno (percloroetileno 0 tetracloroetileno) y tricloroeteno, la suma de concentraciones no debe superar 0,01 $\mathrm{mg} / \mathrm{L}$.

f Trihalometanos: la suma de los cocientes de la concentración de cada compuesto entre sus respectivos valores de referencia y que no debe ser mayor que 1.

${ }^{9} \mathrm{~Bq}:$ becquerel; unidad derivada del Sistema Internacional de Unidades que mide la actividad radiactiva.

${ }^{\mathrm{h}} \mathrm{pCi} / \mathrm{L}=$ picocurios/litro.

mente mayor de cáncer cerebral en su descendencia.

\section{Derivados del petróleo}

Un estudio ecológico realizado en un pueblo de Ecuador, que estaba rodeado de campos de petróleo e importantes empresas petrolíferas (24) y cuya $\mathrm{ACH}$ presentaba concentraciones de hidrocarburos de hasta 2,88 mg/L, reveló un aumento en la incidencia de casos de cáncer, independientemente de su tipo. Entre los diez casos de cáncer diagnosticados, había un niño de 5 años con leucemia. Sin embargo, no se encontró asociación significativa de la expo- sición a hidrocarburos con la presencia de cáncer.

\section{Plaguicidas agrícolas y herbicidas}

Tres estudios llevados a cabo en los Estados Unidos $(14,17,19)$ evaluaron la contaminación por distintos compuestos químicos no permitidos en el ACH. En uno de ellos (14), realizado en 208 cuencas hidrográficas del norte de Texas, se valoró el riesgo para distintos tipos de cáncer infantil en relación con la exposición desde el nacimiento a la contaminación con plaguicidas agrícolas arsenicales y herbicidas a través del $\mathrm{ACH}$; se encontró que había un mayor riesgo en ocho de las cuencas investigadas. En el segundo estudio (17), se evaluó la asociación entre la exposición a herbicidas (atrazina y metolaclor) y cuatro tipos de cáncer infantil (óseo, cerebral, leucemia y linfoma) y se encontró una posible asociación entre la exposición al metolaclor en el ACH y el aumento de casos de cáncer de hueso. Por último, un estudio que evaluó la exposición a otro herbicida (perclorato de amonio) (19) no halló diferencias entre lo observado (38 casos) y la incidencia esperada en la población estudiada para cualquier tipo de cáncer, en residentes menores de 15 años.

\section{Radionúclidos}

En el estudio realizado en Fallon (18), se analizó también la presencia en el $\mathrm{ACH}$ de dos conocidos carcinógenos: el radón $\left({ }^{222} \mathrm{Rn}\right)$ y los isótopos radioactivos de uranio. Sin embargo, no se observó que la concentración de estos compuestos en el agua de pozo de las familias con casos de leucemia infantil difiriera significativamente de la del resto de los pozos domésticos investigados.

En cuatro estudios revisados se analizó la contaminación del ACH con radio ( $\left.{ }^{226} \mathrm{Ra}\right)$. En dos estudios de casos y controles $(27,29)$ realizados en Ontario, Canadá, se concluyó que había una asociación entre el riesgo de padecer osteosarcoma y la exposición al ACH contaminada con este elemento en el lugar de nacimiento. Posteriormente, y mediante un análisis combinado de los participantes en ambos estudios, no se encontró una asociación significativa entre la exposición a lo largo de la vida y el riesgo estudiado. No obstante, la exposición observada en el primero de esos estudios mostró una relación dosis-respuesta significativa en el período de exposición (29). Por otro lado, dos estudios, uno llevado a cabo en el Reino Unido (32) y otro en los Estados Unidos (33), ofrecen resultados contradictorios respecto a la mortalidad local por tumor óseo.

\section{Contaminación de origen industrial y humano}

En un estudio realizado en California, Estados Unidos (19), se evaluó la asociación entre la exposición prolongada al tricloroetileno a través del ACH y el aumento de casos con distintos tipos de cáncer observados en residentes de la zona, incluidos menores de 15 años. El total encontrado de 38 casos, entre ellos 
10 de leucemia infantil y 6 de tumores cerebrales o del sistema nervioso, no difería de lo esperado en la población estudiada para todos los tipos de cáncer combinados.

Por su parte, en un estudio llevado a cabo en el Reino Unido (25) se observó que había un mayor riesgo de muerte antes de los 16 años por cáncer infantil incluida la leucemia- en los nacidos en lugares con exposición a efluentes tóxicos de incineradores y el tiempo de vida en ese lugar. Sin embargo, no se observó ninguna asociación con la exposición a efluentes de vertederos de desechos tóxicos que contaminaban los suelos de la zona rodeados por canales.

En un estudio realizado en New Jersey, Estados Unidos (26), no se pudo constatar si los compuestos de dinitrotetralina, ya fueran trímeros o los productos de su hidrólisis, fueron los responsables de los casos de cáncer infantil diagnosticados en la zona.

Por su parte, en dos estudios ubicados en una zona industrial de Massachusetts, Estados Unidos, se evaluó la posible asociación entre la contaminación del $\mathrm{ACH}$ y la leucemia infantil. En el primero de ellos (30), se concluyó que dos de los ocho pozos de ACH estaban contaminados con tricloroetileno, tetracloroetileno, triclorotrifluoroetano y dicloroetileno y se observó una elevada tasa de casos de leucemia infantil vinculada temporal y espacialmente con esa contaminación. No obstante, en un estudio de casos y controles posterior (20) no se encontró una asociación significativa entre la exposición al agua contaminada y el diagnostico de leucemia durante el embarazo, aunque se demostró una relación dosis-respuesta significativa en este período.

En resumen, la mayoría de los estudios no hallaron una asociación significativa entre la exposición a los contaminantes encontrados en el ACH y el aumento del número de casos de neoplasias malignas (16, 18, 21-24). No obstante, en algunas de las poblaciones estudiadas se observó una relación significativa dosisrespuesta en el período de exposición $(20,29)$.

\section{DISCUSIÓN}

De la revisión realizada se desprende que una gran cantidad de sustancias químicas contaminantes y potencialmente tóxicas se encuentran dispersas en el medio ambiente y se ingieren con el agua. Asimismo, hay consenso en que los niños, desde el nacimiento hasta el final de la adolescencia, son más vulnerables a los contaminantes químicos que los adultos. Esta susceptibilidad durante la infancia - junto con la sensibilidad biológica como característica inherente al crecimiento- puede producir daños irreparables en el desarrollo de sistemas y órganos que pueden llevar a la discapacidad e incluso a la muerte en edades tempranas $(37,38)$. Una cuestión todavía no resuelta es en qué medida los contaminantes químicos dispersos en el medio ambiente pueden estar contribuyendo al cambio de patrón en las enfermedades pediátricas (37).

Queda de manifiesto que el arsénico es el contaminante químico del $\mathrm{ACH}$ más documentado, por su amplia distribución. Las aguas subterráneas son su principal vía de diseminación en el medio ambiente. Sus compuestos inorgánicos, los más tóxicos, se acumulan en algunos acuíferos de forma natural, por la disolución de algunos minerales, como un subproducto de procesos químicos, como resultado de la actividad industrial, por el uso de plaguicidas o por la precipitación atmosférica. Pero, aunque la exposición al arsénico inorgánico por ingesta de agua suele ser pequeña, existen regiones donde se ha comprobado que la contaminación del ACH con este metaloide puede alcanzar niveles dañinos para la salud de la población expuesta (39).

La consecuencia más grave para la salud de la exposición crónica al arsénico estriba en el riesgo de desarrollar cáncer. Este proceso puede demorar entre 5 y 40 años, en dependencia de varios factores, como la susceptibilidad individual, la magnitud y el tiempo de exposición, e incluso las condiciones nutricionales de las personas $(40,41)$. Por esa razón es importante, además de atender a los pacientes que ya han enfermado, poder identificar y ofrecer medidas profilácticas a las personas susceptibles a enfermar en el futuro como consecuencia de una exposición pasada y persistente al arsénico.

El mayor riesgo de enfermedad por exposición al arsénico está relacionado con el consumo de agua contaminada y generalmente ocurre por la ingestión prolongada de agua con bajas concentraciones de arsénico inorgánico (41). Aproximadamente 1 de cada 100 per- sonas que beben agua que contiene 0,05 mg/L de arsénico o más durante largos períodos pueden morir de cáncer provocado por el arsénico (42). Es posible reducir la exposición mediante el tamizaje de las fuentes de abastecimiento de $\mathrm{ACH}$ y el cierre de las que presenten concentraciones de arsénico superiores a 0,01 mg/L (según las recomendaciones de la Organización Mundial de la Salud) o superiores a los límites nacionales permitidos; es necesario, además, llevar a cabo paralelamente campañas de concientización de la población $(41,42)$.

Por otro lado, el nitrato es el contaminante químico más frecuentemente encontrado en el agua subterránea en todo el mundo (43). El consumo de agua contaminada con nitratos es un factor de riesgo de varios tipos de cáncer. Si bien, la Agencia para la Protección Ambiental (EPA), de los Estados Unidos, encontró datos contradictorios sobre la asociación entre la exposición a nitratos o nitritos y el cáncer infantil $(44,45)$, hay datos que apuntan hacia una posible relación entre el contenido elevado de nitratos en el ACH y el cáncer gastrointestinal (46).

También los plaguicidas pueden causar leucemia en niños. Hay informes científicos que indican que los niños con este tipo de cáncer tenían una probabilidad tres veces mayor de que su madre hubiera estado expuesta a plaguicidas durante el embarazo o la lactancia (46).

Otro de los problemas detectados en la revisión es el uso del cloro como desinfectante del ACH. Si bien este método de desinfección soluciona un problema de contaminación biológica, los productos generados durante la desinfección con cloro pueden estar relacionados con la aparición de casos de cáncer de recto y de vejiga; no obstante, esa relación no ha sido estadísticamente significativa hasta el momento (47).

$\mathrm{Al}$ analizar la relación entre la contaminación del ACH y el aumento del número de casos de diferentes tipos de cáncer, se deben tener en cuenta también otras sustancias químicas que no son objeto de búsqueda activa y no se encuentran en las bibliotecas espectrales, o incluso pueden ser productos de la transformación de contaminantes originalmente introducidos en el medio ambiente (48).

$\mathrm{Al}$ analizar estos resultados se debe tener en cuenta que se encontró poca información sobre cáncer infantil por contaminación química del ACH. La ma- 
yoría de la información disponible provenía de estudios ecológicos y en los que las medidas de la exposición podrían no ser precisas o estar sujetas a errores de medición (algunos estudios solamente se refieren a "altas tasas"). Además, en los estudios más recientes se utilizaron datos de mortalidad, pero estos resultados pueden no ser representativos de todos los casos de cáncer diagnosticados en un período específico de tiempo. Por otra parte, aunque las revisiones sistemáticas se deben basar en estudios con seguimientos y diseños que garanticen el mayor rigor científico, en el presente análisis se incluyeron todos los artículos que trataron el tema analizado.

A pesar de esas limitaciones, se puede concluir que a partir de los estudios revisados no se puede afirmar que haya una clara asociación entre la exposición a los contaminantes encontrados en el ACH y el aumento de casos de cáncer en menores de 19 años. No obstante, en algunas poblaciones estudiadas se encontró una relación significativa dosis-respuesta en el período de exposición estudiado.

Teniendo en cuenta el insuficiente nivel de actualidad de los artículos encontrados, se requiere realizar estudios comunitarios encaminados a conocer el efecto real de la contaminación del $\mathrm{ACH}$ en la incidencia de cáncer en la población, en particular en los niños y jóvenes por su mayor susceptibilidad.

Se debe resaltar la necesidad, ya aceptada universalmente, de mejorar el acceso de la población al ACH libre de contaminación. Las autoridades sanitarias y políticas deben darle a este problema de salud pública la máxima prioridad, con lo que se evitará que miles de personas enfermen cada año por beber agua contaminada.

\section{REFERENCIAS}

1. Argos M, Kalra T, Rathouz PJ, Chen Y, Pierce B, Parvez F, et al. Arsenic exposure from drinking water, and all-cause and chronic-disease mortalities in Bangladesh (HEALS): a prospective cohort study. Lancet. 2010;376(9737): 252-8.

2. Fagliano J, Berry M, Bove F, Burke T. Drinking water contamination and the incidence of leukemia: an ecologic study. Am J Public Health. 1990;80(10):1209-12.

3. Cohn P, Klotz J, Bove F, Berkowitz M, Fagliano J. Drinking water contamination and the incidence of leukemia and non-Hodgkin's lymphoma. Environ Health Perspect. 1994;102(6-7): 556-61.

4. Preston JR. Children as a sensitive subpopulation for the risk assessment process. Toxicol Appl Pharmacol. 2004;199(2):132-41.

5. International Agency of Research on Cancer. Monographs on the evaluation of carcinogenic risk to humans. Vol. 84. Some drinking-water disinfectants and contaminants, including arsenic. Lyon: World Health Organization; 2004.

6. Cantor KP. Drinking water and cancer. Cancer Causes Control. 1997;8(3):292-308.

7. United Nations Environment Programme, International Labour Organization, World Health Organization. Environmental health criteria 224: arsenic and arsenic compounds. Geneva: WHO; 2001.

8. Villanueva CM, Grimalt JO, Ballester F, Ibarluzea J, Sala M, Tardón A, et al. Medida de contaminantes del agua y usos del agua durante el embarazo en un estudio de cohortes en España. Gac Sanit. 2006;20(Supl 3):1-9.

9. Ward MH, de Kok TM, Levallois P, Brender J, Gulis G, Nolan BT, et al. Workgroup report: drinking water nitrate and health-recent findings and research needs. Environ Health Perspect. 2005;113(11):1607-14.

10. Infante-Rivard $C$. Chemical risk factors and childhood leukaemia: a review of recent studies. Radiat Prot Dosimetry. 2008;132(2): 220-7.

11. Von Elm E, Altman DG, Egger M, Pocock SJ, Gøtzsche PC, Vandenbroucke JP. Declaración de la Iniciativa STROBE: directrices para la comunicación de estudios observacionales. Gac Sanit. 2008;22(2):144-50. Disponible en: http://www.strobe-statement.org/ Acceso el 9 de diciembre de 2012.

12. Wanden-Berghe C, Sanz-Valero J. Systematic reviews in nutrition: standardized methodology. Br J Nutr. 2012;107(Suppl 2):S3-7.

13. López Piñero JM, Terrada ML. Los indicadores bibliométricos y la evaluación de la actividad médico-científica (II). La comunicación científica en las distintas áreas de las ciencias médicas. Med Clin (Barcelona). 1992;98(3):101-6.

14. Thompson JA, Carozza SE, Bissett WT, Zhu L. Risks of childhood cancer among Texas watersheds based on mothers' living locations at the time of birth. J Water Health. 2010;8(1):139-46.

15. Liaw J, Marshall G, Yuan Y, Ferreccio C, Steinmaus C, Smith AH. Increased childhood liver cancer mortality and arsenic in drinking water in northern Chile. Cancer Epidemiol Biomarkers Prev. 2008;17(8):1982-7.

16. Mukherjee SC, Saha KC, Pati S, Dutta RN, Rahman MM, Sengupta MK, et al. Murshidabad-one of the nine groundwater arsenicaffected districts of West Bengal, India. Part II: dermatological, neurological, and obstetric findings. Clin Toxicol (Phila). 2005;43(7): $835-48$.

17. Thorpe N, Shirmohammadi A. Herbicides and nitrates in groundwater of Maryland and childhood cancers: a geographic information systems approach. J Environ Sci Health C Environ Carcinog Ecotoxicol Rev. 2005;23(2): 261-78.

18. Seiler RL. Temporal changes in water quality at a childhood leukemia cluster. Ground Water. 2004;42(3):446-55.

19. Morgan JW, Cassady RE. Community cancer assessment in response to long-time exposure to perchlorate and trichloroethylene in drinking water. J Occup Environ Med. 2002;44(7):616-21.
20. Costas K, Knorr RS, Condon SK. A case-control study of childhood leukemia in Woburn, Massachusetts: the relationship between leukemia incidence and exposure to public drinking water. Sci Total Environ. 2002;300 (1-3):23-35.

21. Moore LE, Lu M, Smith AH. Childhood cancer incidence and arsenic exposure in drinking water in Nevada. Arch Environ Health. 2002;57(3):201-6.

22. Infante-Rivard C, Olson E, Jacques L, Ayotte P. Drinking water contaminants and childhood leukemia. Epidemiology. 2001;12(1): 13-9.

23. Mueller BA, Newton K, Holly EA, PrestonMartin S. Residential water source and the risk of childhood brain tumors. Environ Health Perspect. 2001;109(6):551-6.

24. San Sebastián M, Armstrong B, Córdoba JA, Stephens C. Exposures and cancer incidence near oil fields in the Amazon basin of Ecuador. Occup Environ Med. 2001;58(8):517-22.

25. Knox EG. Childhood cancers, birthplaces, incinerators and landfill sites. Int J Epidemiol. 2000;29(3):391-7.

26. Richardson SD, Collette TW, Price PC, Genicola FA, Jenks JW, Thruston AD, et al Identification of drinking water contaminants in the course of a childhood cancer investigation in Toms River, New Jersey. J Expo Anal Environ Epidemiol. 1999;9(3):200-16.

27. Finkelstein MM, Kreiger N. Radium in drinking water and risk of bone cancer in Ontario youths: a second study and combined analysis. Occup Environ Med. 1996;53(5):305-11.

28. Tsuda T, Babazono A, Yamamoto E, Kurumatani N, Mino Y, Ogawa T, et al. Ingested arsenic and internal cancer: a historical cohort study followed for 33 years. Am J Epidemiol. 1995;141(3):198-209.

29. Finkelstein MM. Radium in drinking water and the risk of death from bone cancer among Ontario youths. Can Med Assoc J. 1994;151(5):565-71.

30. Lagakos SW, Wessen BJ, Zelen M. An analysis of contaminated well water and health effects 
in Woburn, Massachusetts. J Am Stat Assoc. 1986;81(395):583-96.

31. Vidal V, Ochoa A. Chronic arsenic poisoning. Bol Med Hosp Infant Mex. 1979;36(5):849-61.

32. Boyd JT, Doll R, Hill GB, Sissons HA. Mortality from primary tumours of bone in England and Wales, 1961-63. Br J Prev Soc Med. 1969;23(1):12-22.

33. Petersen NJ, Samuels LD, Lucas HF, Abrahams SP. An epidemiologic approach to low-level radium 226 exposure. Public Health Rep. 1996;81(9);805-14.

34. Sanderson S, Tatt ID, Higgins JPT. Tools for assessing quality and susceptibility to bias in observational studies in epidemiology: a systematic review and annotated bibliography. Int J Epidemiol. 2007;36(3):666-76.

35. Miralles J, Ramos JM, Ballester R, Belinchon I, Sevilla A, Marangon M. Estudio bibliométrico de la revista Actas Dermo-Sifiliográficas (19842003). II. Análisis de las referencias bibliográficas. Actas Dermosifiliogr. 2005;96(9):563-71.

36. Casterá VT, Sanz-Valero J, Juan-Quilis V, Wanden-Berghe C, Culebras JM, García de Lorenzo y Mateos A. Estudio bibliométrico de la revista Nutrición Hospitalaria en el período 2001 a 2005: parte II, análisis de consumo; las referencias bibliográficas. Nutr Hosp. 2008;23(6):541-6.

37. Landrigan PJ, Schechter CB, Lipton JM, Fahs MC, Schwartz J. Environmental pollutants and disease in American children: estimates of morbidity, mortality and costs for lead poisoning, asthma, cancer and development disabilities. Environ Health Perspect. 2002;110(7):721-8.

38. Bearer CF. How are children different from adults? Environ Health Perspect. 1995;103 (Suppl 6):7-12.

39. Smeldey PL, Kinniburgh DG. A review of the source, behaviour, and distribution of arsenic in natural waters. Appl Geochem. 2002;17(5):517-68.

40. Ochoa RJ, Vidal LA, Lerma SA, Gómez RL, Reta SA, Fernández ED, et al. Arsenicismo subagudo y crónico en una población rural. Dermatol Rev Mex. 2009;53(2):63-9.

41. World Health Organization. Guidelines for drinking-water quality. Vol. 1. Recommendations. 3th ed. Geneva: WHO; 2008.

42. Smith AH, Lingas EO, Rahman M. Contamination of drinking-water by arsenic in Bangladesh: a public health emergency. Bull World Health Org. 2000;78(9):1093-103.

43. Spalding RF, Exner ME. Occurrence of nitrate in groundwater - a review. J Environ Qual. 1993;22(3):392-402.

44. United States Environmental Protection Agency. Six-year review, chemical contaminants health effects: technical support document. Washington, D.C.: US-EPA; 2003. Disponible en: http://water.epa.gov/lawsregs/ rulesregs/regulatingcontaminants/sixyear review/first_review/upload/support_6yr healtheffects_final.pdf Acceso el 25 mayo de 2012.
45. United States Environmental Protection Agency. Guidelines for carcinogen risk assessment (EPA/630/P-03/001F). Washington, D.C.: US-EPA; 2005. Disponible en: http:// www.epa.gov/raf/publications/pdfs/can cer guidelines final 3-25-05.pdf Acceso el 21 de mayo de 2012.

46. Quintero-Somaini A, Quirindongo M. Peligro oculto, riesgos ambientales a la salud de la comunidad latina. New York, NY: Natural Resources Defense Council; 2004.

47. Morris RD, Audet AM, Angelillo IF, Chalmers TC, Mosteller F. Chlorination, chlorination byproducts, and cancer: a meta-analysis. Am J Public Health. 1992;82(7):955-63.

48. United States Environmental Protection Agency. Second five-year review for Reich Farm Superfund Site, Toms River, Ocean County, New Jersey. Washington, D.C.: US-EPA; 2005. Disponible en: http:// www.epa.gov/superfund/sites/fiveyear/ f2008020002583.pdf Acceso el 25 de mayo de 2012.

Manuscrito recibido el 25 de noviembre de 2011. Aceptado para publicación, tras revisión, el 9 de septiembre de 2012

ABSTRACT Objective. To evaluate the association between exposure to the main chemical contaminants in drinking water and the rise in cancer cases among the population under age 19.

Cancer in the population under 19 years of age caused by chemical contamination in drinking water: a systematic review
Methods. A systematic review was undertaken of the scientific literature compiled in the MEDLINE (via PubMed ${ }^{\odot}$ ), EMBASE $^{\odot}$, Web of Knowledge, Cochrane Library Plus, Latin American and Caribbean Literature on Health Sciences (LILACS), SCOPUS, and SCIRUS databases. The descriptors used were "neoplasms" and "water pollution, chemical," limited to studies that included people under age 19. Articles selected were of any type in any language, from the inception of the indexing of the primary source until March of 2011.

Results. The search generated 266 articles, from which 20 were selected after applying the inclusion and exclusion criteria. Drinking water contaminants analyzed were arsenic, disinfection byproducts, nitrogen compounds, petroleum derivatives, agricultural pesticides, radionuclides, and others of industrial origin. The majority of the studies did not find a significant link between exposure to drinking water contaminants and the increase in cancer cases in the under-19 population segment. In some of the studied populations a significant dose-response relationship was observed.

Conclusions. Taking into account that the articles located were insufficiently upto-date, more studies are required in order to know the effect of drinking water contamination on cancer rates, in particular among children and youths, who are more susceptible.

Key words Neoplasms; water pollution, chemical; child; adolescent; drinking water. 